\title{
Numerical Solution of a Two-Particle Model of Positronium Confined in Small Cavities
}

\author{
G. Tanzi Marlottit ${ }^{a}$, F. Castelli ${ }^{a, b}$ And G. Consolati ${ }^{c, b}$ \\ ${ }^{a}$ Department of Physics, Università degli Studi di Milano, via Celoria 16 I-20133 Milano, Italy \\ ${ }^{b}$ INFN, sezione di Milano, via Celoria 16 I-20133 Milano, Italy \\ ${ }^{c}$ Department of Aerospace Science and Technology, Politecnico di Milano, via LaMasa 34, I-20156 Milano, Italy
}

\begin{abstract}
The positronium atom (Ps) is widely used as a probe to characterize nanoporous and mesoporous materials. Existing theoretical models for describing Ps annihilation rates by pick-off processes generally treat Ps as a point particle confined in a potential well. Hence these models do not justify any change in the internal structure of Ps, which is experimentally accessible by means of the contact density parameter. Recently we formulated a twoparticle model in which only the electron is confined in the cavity, while the positron is moving freely and feels the medium via a positive work function. We present here a numerical treatment of the problem of calculating contact densities and pick-off annihilation rates, by using a variational method. Results are in agreement with experimental data for a large class of materials, and suggest a way to connect these data with pore sizes and positron work functions.
\end{abstract}

DOI: $10.12693 /$ APhysPolA.132.1575

PACS/topics: 36.10.Dr, 34.80.Lx, 78.70.Bj

\section{Introduction}

Positronium (Ps), the hydrogen-like bound state of an electron and a positron, has a widespread use as a probe for studying nanoscale structures in condensed matter, thanks to its annihilation properties. As a matter of fact, after formation (by implanting positrons) Ps can be found into nanometric cavities where the lifetime of the long-living component $o$-Ps (142 ns) is strongly reduced (until a few ns) by pick-off annihilation of the positron with an electron of the surrounding cavity walls [1]. While in vacuum the annihilation rate depends on the electron density at the positron position (the contact density [2]), pick-off processes, which dominates the annihilation in small cavities, depend on the electron density in the bulk around the cavity. Experimental data obtained with positron annihilation lifetime spectroscopy (PALS) concern pick-off annihilation lifetimes and contact densities [3]. By connecting these data with suitable models of the properties of Ps in small cavities, it would be possible to obtain information on pore dimensions and other material characteristics, like the work functions.

To describe Ps inside small cavities the most used models are based on the Tao-Eldrup approach [4], which relates pick-off annihilation rates $\lambda_{\text {ckoff }}$ to pore sizes by considering Ps as a single quantum particle inside an potential well. However, by definition, this assumption gives no information about contact densities. Another family of models consider both Ps constituent particles as independent but confined into the cavity $[5,6]$, or interacting with the material through effective potentials [7], or finally forming a bubble-like state in a dielectric medium with special assumption about work functions [8]. Only the last model can describe the wellknown observation that the contact density is usually found to be well below the vacuum value, while all other models predict an increase. Therefore these models are not fully satisfactory. The reason for this may be recognized in the observation that the confining potential acting on Ps is a net result of two independent and different contributions, acting on the electron and on the positron separately. In particular, a positive value for the positron work function, as derived by theoretical models [9] and found, for example, in silica [10], suggests that the positron is attracted toward the medium and then is not confined a priori. On the other hand, the strong repulsive Pauli exchange forces with bulk electrons are expected to exert a confinement effect on the electron [11]. Starting from these observations, we reconsidered the problem of Ps in nanopores, and have proposed [12] a two-particle model with appropriate potentials, different for each constituent particle. By applying approximate semi-analytical techniques, we were able to demonstrate that our model correctly describe the lowering of the contact density, obtaining also promising results in the comparison with experimental data on this quantity and on the pick-off annihilation rate, connected with the pore radius and the positron work function. Here we make a step further by exploring the potentiality of our twoparticle model with a numerical approach for deriving the relevant quantities.

\section{Theory}

Assuming for definiteness a spherical cavity of radius $R_{c}$ centered on the axes origin we write the Hamiltonian operator for a system of 2 particles with the same mass $m$ and opposite charges $\pm e$ subjected to different potentials

$$
H=\frac{p_{1}^{2}}{2 m}+\frac{p_{2}^{2}}{2 m}-\frac{e^{2}}{\left|\boldsymbol{q}_{1}-\boldsymbol{q}_{2}\right|}+V_{\text {conf }}\left(q_{1}\right)+V_{\text {bulk }}\left(q_{2}\right),(1)
$$

where $\boldsymbol{q}_{1}\left(\boldsymbol{q}_{2}\right)$ is the position of the confined electron 
(positron). $V_{\text {conf }}$ and $V_{\text {bulk }}$ describe the interaction with the surrounding medium. The confining potential acts only on the electron and can be taken as a well with infinite depth

$$
V_{\text {conf }}\left(q_{1}\right)=\left\{\begin{array}{lll}
0 & \text { if } & \left|\boldsymbol{q}_{1}\right|<R_{c}, \\
\infty & \text { if } & \left|\boldsymbol{q}_{1}\right|>R_{c} .
\end{array}\right.
$$

On the other hand, the bulk potential acts only on the positron and in the first approximation can be taken as equal to the opposite of the positron work function $\phi_{+}$ deep inside the bulk, and zero in the electron-confining cavity

$$
V_{\text {bulk }}\left(q_{2}\right)=\left\{\begin{array}{lll}
0 & \text { if } & \left|\boldsymbol{q}_{2}\right|<R_{c} \\
-\phi_{+} & \text {if } & \left|\boldsymbol{q}_{2}\right|>R_{c}
\end{array}\right.
$$

This way we assume that the positron is attracted to the bulk and perceives a free volume cavity as a potential barrier. Since the bulk potential has to be considered small (in fact of the order of a few $\mathrm{eV}$ at most [10]) the system is dominated by the confining and the Coulombic potentials. Bearing this in mind, it is convenient to perform a non-standard change of coordinates that is more suitable for the present situation

$$
\left\{\begin{array} { l } 
{ \boldsymbol { r } = \boldsymbol { q } _ { 2 } - \boldsymbol { q } _ { 1 } } \\
{ \boldsymbol { p } = \boldsymbol { p } _ { 2 } }
\end{array} \quad \left\{\begin{array}{l}
\boldsymbol{r}_{e}=\boldsymbol{q}_{1} \\
\boldsymbol{P}=\boldsymbol{p}_{1}+\boldsymbol{p}_{2}
\end{array},\right.\right.
$$

which satisfy the usual commutation relations. Here $\boldsymbol{r}_{e}$ is again the confined electron position, while $\boldsymbol{r}$ represents the relative distance with the positron. With this variables the Hamiltonian operator in Schrödinger representation becomes (in atomic units)

$$
\begin{aligned}
H & =-\frac{1}{2} \nabla_{r_{e}}^{2}-\nabla_{r}^{2}+\nabla_{r_{e}} \cdot \nabla_{r}-\frac{1}{r}+V_{\text {conf }}\left(r_{e}\right) \\
& +V_{\text {bulk }}\left(\boldsymbol{r}_{e}, \boldsymbol{r}\right)=H_{0}+V_{\text {mix }},
\end{aligned}
$$

where we have defined the mixing potential

$$
V_{\text {mix }}=\nabla_{r_{e}} \cdot \nabla_{r}+V_{\text {bulk }}\left(\boldsymbol{r}_{e}, \boldsymbol{r}\right) \text {. }
$$

It can be easily shown that the Hamiltonian $H_{0}$, which contains both the $V_{\text {conf }}$ and the Coulombic potential, is separable and has exact solutions. For the ground state, one has $E_{0}=\frac{\pi^{2}}{2 R_{c}^{2}}-\frac{1}{4}$ and

$$
\Psi_{0}\left(\boldsymbol{r}_{e}, \boldsymbol{r}\right)=\frac{1}{N} \frac{\sin \left(\pi r_{e} / R_{c}\right)}{r_{e}} \exp \left(-\frac{r}{2}\right) .
$$

Given the spherical symmetry, the problem is symmetric both with respect to rotation around the direction of $\boldsymbol{r}_{e}$ and with respect to a rigid rotation of both the particles around the center of the cavity. In particular it is invariant under the inversion operator $I:\left(\boldsymbol{r}, \boldsymbol{r}_{e}\right) \rightarrow$ $\left(-\boldsymbol{r},-\boldsymbol{r}_{e}\right)$ which preserves the angle $\alpha$ between the two vectors $\boldsymbol{r}_{e}$ and $\boldsymbol{r}$. From these considerations, it seems natural to search for a ground state wave function which depends only on the absolute values $r, r_{e} \in\left[0 ; R_{c}\right]$ and on the angle $\alpha\left(\boldsymbol{r}, \boldsymbol{r}_{e}\right) \in[0 ; \pi]$ between them. By using these variables, the new Hamiltonian of the system assumes the form

$$
\tilde{H}=-\frac{1}{2}\left[\nabla_{r_{e}}^{2}+2 \nabla_{r}^{2}+\frac{2 r_{e}^{2}+r^{2}+2 r r_{e} \cos \alpha}{r^{2} r_{e}^{2}} \nabla_{\alpha}^{2}\right.
$$

$$
\begin{aligned}
& -2 \cos \alpha \nabla_{r_{e}, r}^{2}+2 \frac{\sin \alpha}{r} \nabla_{r_{e}, \alpha}^{2}+2 \frac{\sin \alpha}{r_{e}} \nabla_{r, \alpha}^{2} \\
& \left.+\frac{2}{r_{e}} \nabla_{r_{e}}+\frac{4}{r} \nabla_{r}+\frac{2 r_{e}^{2} \cos \alpha+r^{2} \cos \alpha+2 r r_{e}}{r^{2} r_{e}^{2} \sin \alpha} \nabla_{\alpha}\right] \\
& -\frac{1}{r}+V_{\text {conf }}\left(r_{e}\right)+V_{\text {bulk }}\left(r, r_{e}, \alpha\right)=H_{\text {conf }}+V_{\text {bulk }},
\end{aligned}
$$

where $H_{\text {conf }}$ include both the confining and Coulombic potential.

Calculation of the relative contact density requires the knowledge of the two-particle ground state wave function $\Psi_{G S}\left(r_{e}, r, \alpha\right)$ in $\boldsymbol{r}=0$, integrated over the electron position in the cavity [12]:

$$
k_{r}=4 \int_{0}^{R_{c}}\left|\Psi_{G S}\left(r_{e}, 0,0\right)\right|^{2} r_{e}^{2} \mathrm{~d} r_{e} .
$$

To numerically solve the full Schrödinger equation of the system we used the Ritz variational method to find an approximated ground state wave function. The method consists of choosing a basis set of parameter-dependent trial wave functions $\Psi_{n}[\epsilon]$, and finding the linear combination $\tilde{\Psi}=\sum_{n} C_{n} \Psi_{n}[\epsilon]$ for which the expectation value of the energy $\langle\tilde{\Psi}|\tilde{H}| \tilde{\Psi}\rangle$ is the lowest possible.

Following Fülöp et al. [11] and inspired by the form of the wave functions of $H_{0}$ (see Eq. (7)) we introduce the following ansatz for the basis set:

$$
\Psi_{i j k}[\epsilon]\left(r_{e}, r, \alpha\right)=\psi_{i}^{(1)}\left(r_{e}\right) \cdot \psi_{j}^{(2)}(r) \cdot \psi_{k}^{(3)}(\alpha) .
$$

The three components are given by

$$
\begin{aligned}
& \psi_{i}^{(1)}\left(r_{e}\right)=\left(R_{c}^{2}-r_{e}^{2}\right)^{i}, i=\left(1, \cdots, N_{1}\right), \\
& \psi_{j}^{(2)}(r)=r^{j-1} e^{-r / \epsilon}, j=\left(1, \cdots, N_{2}\right), \\
& \psi_{k}^{(3)}(\alpha)=\cos ^{k-1} \alpha, k \leq j,
\end{aligned}
$$

where $N_{1}$ and $N_{2}$ determine the size of the basis expansion while the condition $k \leq j$ must be present in order to make the wave function single-valued everywhere. Here $\epsilon$ is an additional adjustable parameter, representing the possibility of a homogeneous swelling of the Bohr Ps radius (for free Ps, $\epsilon=2 a_{B}$ ), and with respect to which the energy is also minimized. Also note that the correct boundary conditions for the electron wave functions are satisfied: $\psi_{i}^{(1)}\left(R_{c}\right)=0$ and $\frac{\mathrm{d} \psi^{(1)}}{\mathrm{d} r_{e}}(0)=0$. These functions are neither normalized nor orthogonal. However, the variational method with minor theoretical modifications can be applied also with a non orthogonal basis set.

Our total trial wave function can be expanded on this basis set

$$
\tilde{\Psi}=\sum_{i}^{N_{1}} \sum_{j}^{N_{2}} \sum_{k \leq j} C_{i j k} \Psi_{i j k}[\epsilon] .
$$

The energy minimization requires the calculation of the Hamiltonian matrix $H_{n n^{\prime}}^{\epsilon}\left\langle\Psi_{i j k}|\tilde{H}| \Psi_{i^{\prime} j^{\prime} k^{\prime}}\right\rangle$ as well as the overlap matrix $S_{n n^{\prime}}^{\epsilon}=\left\langle\Psi_{i j k}\right\rangle \Psi_{i^{\prime} j^{\prime} k^{\prime}}$ for every value of the parameter $\epsilon$. Due to the simple form of the basis functions, $\left(H_{\text {conf }}\right)_{n n^{\prime}}^{\epsilon}$ matrix elements can be calculated analytically, however $\left(V_{\text {bulk }}\right)_{n m}$ must be calculated numerically. 
We performed the analytical calculation with Mathematica 10 and we subsequently evaluated the numerical solution of the eigenproblem with a $\mathrm{C}++$ code. For every set of $\left(R_{c}, \phi_{+}\right)$, the lowest eigenvalue was minimized as a function of $\epsilon$ to obtain the ground-state energy $E_{\mathrm{GS}}$, and the eigenvector $\tilde{\Psi}$ corresponding to this eigenvalue was determined to be the approximate ground-state wave function.

Finally, the relative contact density and the pickoff lifetime was calculated from $\tilde{\Psi}$.

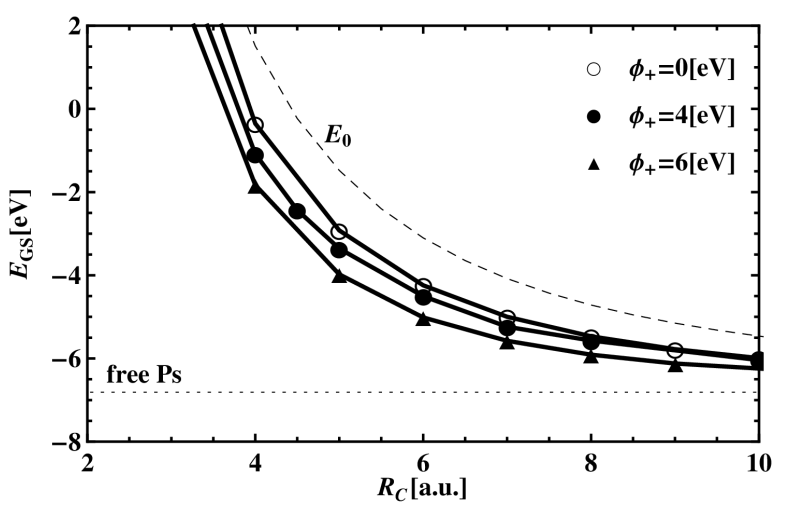

Fig. 1. Ground state energy as a function of the cavity radius $R_{c}$, for different values of the positron work function $\phi_{+}$. The straight dotted line represents the energy of free Ps in vacuum $(-6.8 \mathrm{eV})$ while the dashed line represents the exact ground state energy of $H_{0}$. In the limit $R_{c} \rightarrow \infty$ the energy of free Ps is recovered.

\section{Results}

Here we discuss the obtained results with reference to selected suitable values for the work function and cavity radius. Generally speaking, for the calculation of the energy, a base functions set of $N_{1}=3, N_{2}=3$ was sufficient, while the contact density and the pickoff annihilation rate calculations required more care. For relatively low values of $\phi_{+}$(below $5.9 \mathrm{eV}$ ) a base functions set of $N_{1}=5, N_{2}=5$ already gives good results, in the sense that any further increase of this number has a negligible influence on the calculated values of $E_{\mathrm{GS}}, k_{r}, \lambda_{\text {ckoff. }}$. It turns out that in order to fit almost all available experimental data, this range of values for the positron work function is sufficient. On the other side, for greater values of $\phi_{+}$the behavior of the wave function started to change drastically and the number of basis set needed to reach the same accuracy increase exponentially, making it manifest the need of a modified basis set. Anyway, during our calculations we decide to use a basis set with $N_{1}=6, N_{2}=6$. The resulting ground state energy energy $E_{0}$ is plotted in Fig. 1 as a function of the confining radius $R_{c}$. For large radii, free Ps energy of $-6.8 \mathrm{eV}$ is recovered. The confinement tends to increase the energy with respect to free $\mathrm{Ps}_{\mathrm{s}}$, while the bulk potential has a lesser effect.

The mixing term $V_{\text {mix }}$ in (6) leads to a distortion of the relative wave function of the positron around the electron position. As plotted in Fig. 2, the positron is strongly polarized toward the inner of the cavity via $\nabla_{r_{e}} \cdot \nabla_{r}$, while its attraction toward the outer bulk due to $V_{\text {bulk }}$.
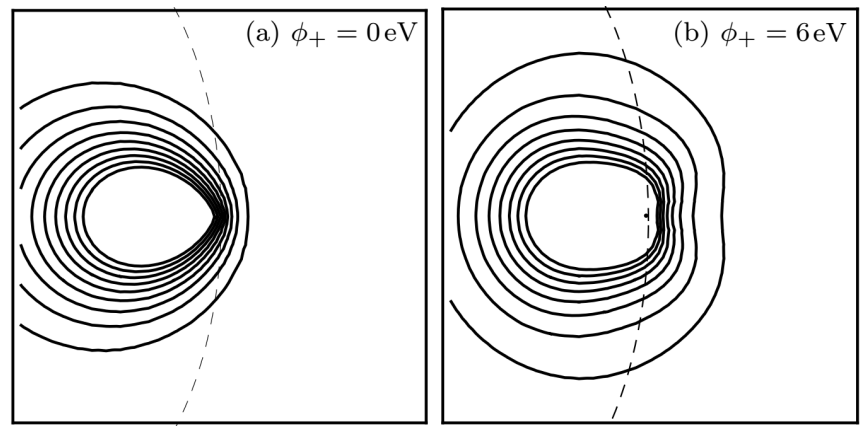

Fig. 2. Polarization of the relative wave function of the positron around the electron, for a cavity $R_{c}=5 \mathrm{a} . \mathrm{u}$. The electron position is fixed at the wall of the cavity, which is located on the left side of both figures.

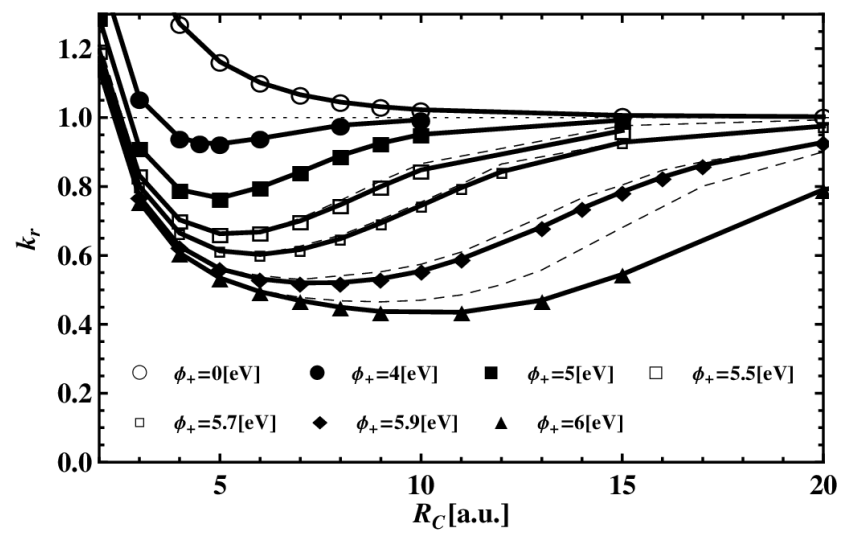

Fig. 3. Relative contact density $k_{r}$ as a function of the confining radius $R_{c}$, for some values of the positron work function $\phi_{+}$. Full lines are calculated with the basis set $N_{1}=6, N_{2}=6$, while the dashed lines are calculated with the basis $N_{1}=5, N_{2}=5$. The distance between those lines can be considered as a raw error estimation.

The resulting relative contact density $k_{r}$ is plotted in Fig. 3 as a function of the confining radius $R_{c}$ and for some values of the positron work function $\phi_{+}$. The effect of lowering of the contact density, with respect to its vacuum value, shows up when $\phi_{+}>3 \mathrm{eV}$. This value is slightly larger than the results found in our previous work [12], but the qualitative trend is recovered. In the point cavity limit $R_{c} \rightarrow 0$ the electron is stuck at the origin and the Schrödinger equation of the hydrogen atom is recovered, where $k_{r} \rightarrow 8$. On the other hand, when $R_{c} \rightarrow \infty$ free Ps solution is recovered, with $k_{r} \rightarrow 1$.

Experimental data for Ps in small cavities usually directly concern the $o$-Ps lifetime $\tau=\frac{1}{\lambda_{\text {ckoff }}}$. By using the Tao-Eldrup approximation [1, 4]:

$$
\lambda_{\text {ckoff }}=2 p_{\text {out }}[\mathrm{ns}]^{-1},
$$

where $\lambda_{\text {ckoff }}$ is the pickoff annihilation rate which dominates the annihilation process in small cavities and which is proportional to the probability of finding the positron 
outside the cavity

$$
p_{\text {out }}=\int_{\left|\boldsymbol{r}_{e}+\boldsymbol{r}\right|>R_{c}}\left|\tilde{\Psi}\left(r_{e}, r, \alpha\right)\right|^{2} r_{e}^{2} r^{2} \sin \alpha \mathrm{d} r_{e} \mathrm{~d} r \mathrm{~d} \alpha .(14)
$$

In Fig. 4 we plot some curves joining points corresponding to calculated values of $k_{r}$ and $\tau$ for a fixed $\phi_{+}$and different $R_{c}$. Known experimental data for some materials are normalized following the discussion in [12] and are indicated by markers.

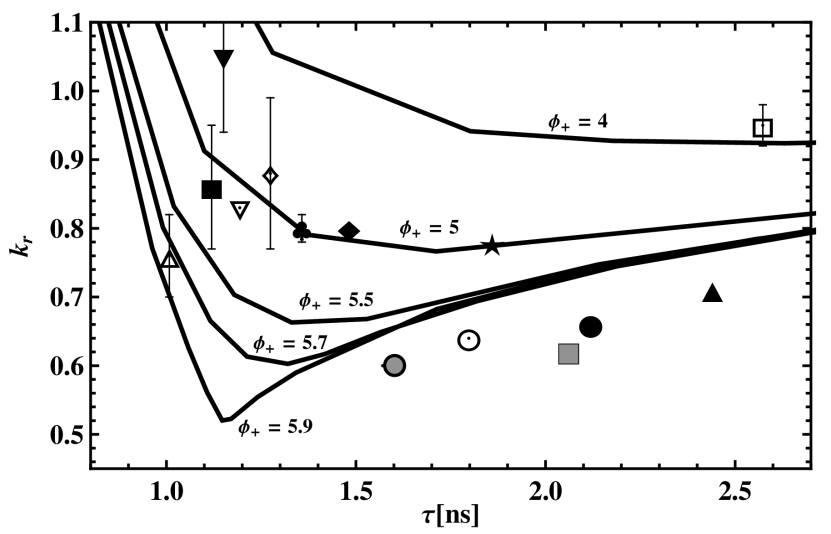

Fig. 4. Relative contact density $k_{r}$ of Ps as a function of its lifetime $\tau$, for different values of the positron work function $\phi_{+}($in $\mathrm{eV})$. Selected lines are calculated with the basis set $N_{1}=6, N_{2}=6$.

It must be noted that this kind of representation is the better choice to compare theoretical and experimental data, because it is very difficult to gain independent information on the positron work function and on the pore sizes for most materials. The general trend shows a lowering of the contact density and of the o-Ps lifetime for smaller cavities, as expected. There is a good agreement between our theory and experimental data for a large group of hydrocarbon molecular solids. On the other hand, some compounds stand below the line $\phi_{+} \sim$ $6 \mathrm{eV}$, probably because their effective electron density is lower than our estimation, so that their position in the picture should be shifted to the left.

\section{Conclusion}

Our two-particle theoretical model describes the internal properties of Ps confined in nanosized cavities. Due to the presence of different boundary conditions and potentials for the two particles (only the electron is strictly confined) a numerical solution is needed. We showed that for a simple geometry a variational approach succeeds in justifying the well known fact of the lowering of the contact density. Measurements of Ps lifetime in solids have a long story as a probe for guessing the size of internal cavities. We showed here how information on this quantity and on the positron work function can be inferred from independent measurements on Ps pick-off annihilation and contact density.

\section{References}

[1] A. Dupasquier, P. DeNatale, A. Rolando, Phys. Rev. $B$ 43, 10036 (1991).

[2] V.I. Goldanskii, At. Energy Rev. 6, 3 (1968).

[3] G. Consolati, F. Quasso, Appl. Phys. A 52, 295 (1991).

[4] S.J.Tao, J. Chem. Phys. 56, 5499 (1972).

[5] G. Consolati, F. Quasso, D. Trezzi, Plos One 9 e109937 (2014).

[6] W. Brandt, S. Berko, W.W. Walker, Phys. Rev. 120, 1289 (1960).

[7] P.A. Sterne, L. Larrimore, P. Hastings, A.L.R. Bug, Radiat. Phys. Chem. 68, 409 (2003).

[8] S.V. Stepanov, D,S. Zvezhinskiy, V.M. Byakov, Mater. Sci. Forum 733, 7 (2013).

[9] M.J. Puska, R.M. Nieminen, Rev. Mod. Phys. 66, 841 (1994).

[10] Y. Nagashima, Y. Morinaka, T. Kurihara, Y. Nagai, T. Hyodo, T. Shirada, K. Nahara, Phys. Rev. B 58, 12676 (1998).

[11] T. Fülöp, Z. Farkas, A. Seeger, J. Major, arXiv:condmat/0304442v1.

[12] G.M. Tanzi, F. Castelli, G. Consolati, Phys. Rev. Lett. 116, 033401 (2016). 